\title{
Chemical reaction rates using the semiclassical Van-Vleck initial value representation
}

\author{
Charulatha Venkataraman and William H. Miller \\ Department of Chemistry and Kenneth S. Pitzer Center for Theoretical Chemistry, \\ University of California, and Chemical Sciences Division, Lawrence Berkeley National Laboratory, \\ Berkeley, California 94720
}

\begin{abstract}
A semiclassical IVR formulation using the Van-Vleck propagator has been used to calculate the flux correlation function and thereby reaction rate constants. This VanVleck formulation of the flux-flux correlation function is computationally as simple as the classical Wigner model. However unlike the latter, it has the ability to capture quantum interference/coherence effects. Classical trajectories are evolved starting from the dividing surface that separates reactants and products, and are evolved negatively in time. This formulation has been tested on model problems ranging from the Eckart barrier, double well to the collinear $\mathrm{H}+\mathrm{H}_{2}$.
\end{abstract}

\section{Introduction}

The calculation of quantum mechanical reaction rates in condensed phase systems has been a challenging problem. Based on linear response theory, any dynamical property of a system close to equilibrium can be expressed in terms of a time correlation function. ${ }^{1,2}$ Miller, Schwartz and Tromp generalized Yamamoto's theory for reaction rates and expressed the reaction rate constant in terms of flux correlation functions. ${ }^{3,4}$

In classical many-body systems, these time correlation functions can be simulated using just Newtonian dynamics. However the calculation of these functions in a quantum system is still a difficult problem.

Basis set methods have been successfully applied for small molecular systems. ${ }^{5}$ However the dimensionality of the basis set scales exponentially with system size. One approach to solving the dimensionality problem is to use path integrals. ${ }^{6}$ But the real time path integrand is too oscillatory and leads to the notorious "sign" problem. ${ }^{7}$ Another approach is to use imaginary time path integral techniques and analytically continue the imaginary time data to real time. ${ }^{8}$ Sophisticated numerical analytic continuation techniques have been designed and used to describe dynamics of complicated systems. ${ }^{9}$ However since these techniques are numerically ill-behaved, these methods are restricted to the description of short time dynamics only and to systems which don't show strong quantum coherences. 
The mixed quantum-classical approach where one treats a few degrees of freedom quantum mechanically and the rest classically, has been quite popular. ${ }^{10}$ In some cases however these methods has been shown to be insufficient to produce accurate rates. ${ }^{11,27}$

Several authors have formulated quantum thermodynamic theories for reaction rates. ${ }^{14}$ Unlike the classical thermodynamic formulation i.e $\mathrm{TST}^{12,13}$ the quantum mechanical version is not unique. An advantage of these formulations is that one needs no real time evolution and can readily use all the imaginary time path integral technology. One such statistical theory, the Quantum Instanton has been shown to give very satisfactory results for a variety of problems ranging from gas phase $\mathrm{H}+\mathrm{CH}_{4}$ reaction to a model for a chemical reaction in a polar solvent. ${ }^{15}$ However as any other transition state theory this method cannot capture recrossing effects and the accuracy of this model depends on the choice of the dividing surface.

Another class of methods which have been shown to reliably substitute exact quantum dynamics are the semiclassical theories. ${ }^{16,17}$ Classical trajectories, which are easy to determine, are at the heart of any semiclassical theory. These theories have been shown to capture quantum interference effects, tunneling etc very accurately; thus they combine the rigor of including quantum effects with the ease of running classical trajectories.

The semiclassical theory based on the $\mathrm{IVR}^{18}$ expresses the quantum time evolution operator as a phase space average over the initial conditions of classical trajectories. Hence the calculation of any semiclassical time correlation function will usually involve a double phase space average as against a single phase space for classical time correlation functions. There is considerable reduction in complexity in using a semiclassical propagator over the exact quantum propagator; however it doesn't completely eliminate the oscillatory nature of the quantum propagator. One thus needs techniques to reduce the oscillation of the semiclassical integrand.

To this end, the Linearized $\operatorname{IVR}^{27,28}$ was introduced; this includes the effects of nearby trajectories only to first order. So this method cannot describe quantum coherence features that arise from distinct classical trajectories, but has been very successful for some model problems. The Forward-Backward Semiclassical dynamics ${ }^{33}$ approach introduced by Makri and Thompson has also been applied to a variety of condensed phase problems. However this model has been shown to be an approximate version of the Linearized IVR and thus cannot describe quantum interference effects beyond the short time limit.

Another idea for evaluating the full SC-IVR expression without invoking the linearization approximation has been the Forward-Backward IVR. ${ }^{29}$ The FB-IVR combines the forward and backward time evolution operators into one semiclassical time propagation; as a result the FB-IVR integrand is considerably less oscillatory. However the FB-IVR is more approximate than the full SC-IVR. The FB-IVR time correlation function is derived by doing one of the integrals in the full SC-IVR time correlation function by a stationary phase approximation. The ability of the FB-IVR to describe interference effects shows a dependance on the observable under consideration. GFB-IVR, which interpolates between the full blown SC-IVR and the FB-IVR, was introduced as a remedy. ${ }^{30}$

The question that we have been asking is: can we get a full blown SC-IVR expression which is only as computationally intensive as the Linearized-IVR, but includes all the quantum interference effects arising from distinct trajectories? In this paper, we have explored this possibility. 
To get such an expression for the time correlation function, we go back to the traditional coordinate state Van-Vleck IVR formulation of the time propagator. Most of the previous rate calculations methods have used the the coherent state IVR of Herman and Kluk. However one knows that the coordinate state matrix element of the Boltzmannized flux operator is much easier to evaluate than the corresponding coherent state matrix element. Due to the presence of the plane wave factor in the coherent state matrix element, the momentum integrand is also more oscillatory. All this may be circumvented by going to the coordinate state representation.

In Sec 2 we describe this Van-Vleck-IVR formulation of the flux correlation function. Even though we have restricted ourselves to flux correlation functions this method can be generalized to any time correlation function. We present the results of some test calculations in Sec 3. The model problems we have tested this method on vary from the one dimensional Eckart barrier to the collinear $\mathrm{H}+\mathrm{H}_{2}$ reaction.

\section{Theory}

The rate constant of a chemical reaction is expressed in terms of a reactive flux correlation function $^{3}$

$$
k(T) Q_{r}(T)=\int_{0}^{\infty} C_{f f}(t) d t,
$$

where $Q_{r}(T)$ is the reactant partition function per unit volume and $C_{f f}(t)$ is the "flux-flux" correlation function,

$$
C_{f f}(t)=\operatorname{tr}\left(\hat{\mathcal{F}}(\beta) e^{i \hat{H} t / \hbar} \hat{F} e^{-i \hat{H} t / \hbar}\right) .
$$

The reactive flux operator is given by

$$
\hat{F}=\frac{i}{\hbar}[\hat{H}, \hat{h}]
$$

where $\hat{H}$ is the Hamiltonian of the system and the projection operator $\hat{h}=h(s(\mathbf{q}))$ is a heaviside function that is 0 (1) on the reactant (product) side of the dividing surface defined by $s(\mathbf{q})=0$. As advocated by Miller, Schwartz and Tromp we employ the split version of the "Boltzmannized" flux operator

$$
\hat{\mathcal{F}}(\beta)=e^{-\beta \hat{\mathcal{H}} / 2} \hat{F} e^{-\beta \hat{\mathcal{H}} / 2}
$$

\subsection{SC-IVR for the flux correlation function}

The semiclassical approximation for the flux correlation function is obtained by using the SC-IVR formalism for the time evolution operator $e^{-i \hat{H} t / \hbar}$. The Van-Vleck-Gutzwiller semiclassical propagator is obtained by evaluating the Feynman propagator by a stationary phase approximation. The IVR version of the propagator removes the cumbersome root search and the singularity present in the original propagator. ${ }^{23}$ The Van-Vleck $(\mathrm{VV})^{19}$ or the coordinate state version of the SC-IVR is given by

$$
e^{-i \hat{H} t / \hbar}=\int d \mathbf{q}_{0} \int d \mathbf{p}_{0} C_{V V}\left(\mathbf{q}_{0}, \mathbf{p}_{0} ; t\right) e^{i S_{t}\left(\mathbf{q}_{0}, \mathbf{p}_{0}\right) / \hbar}\left|\mathbf{q}_{t}\right\rangle\left\langle\mathbf{q}_{0}\right| .
$$


The Herman-Kluk (HK) or the coherent state version of the SC-IVR ${ }^{20,22}$ is given by

$$
e^{-i \hat{H} t / \hbar}=(2 \pi \hbar)^{-f} \int d \mathbf{q}_{0} \int d \mathbf{p}_{0} C_{H K}\left(\mathbf{q}_{0}, \mathbf{p}_{0} ; t\right) e^{i S_{t}\left(\mathbf{q}_{0}, \mathbf{p}_{0}\right) / \hbar}\left|\mathbf{q}_{t} \mathbf{p}_{t}\right\rangle\left\langle\mathbf{q}_{0} \mathbf{p}_{0}\right|
$$

Here $f$ is the number of degrees of freedom, $\left(\mathbf{q}_{0}, \mathbf{p}_{0}\right)$ are the initial coordinates and momenta for a classical trajectory. $\mathbf{q}_{t} \equiv \mathbf{q}_{t}\left(\mathbf{q}_{0}, \mathbf{p}_{0}\right)$ and $\mathbf{p}_{t} \equiv \mathbf{p}_{t}\left(\mathbf{q}_{0}, \mathbf{p}_{0}\right)$ are the variables at time $t$ that evolve from these initial conditions and $S_{t}\left(\mathbf{q}_{0}, \mathbf{p}_{0}\right)$ is the classical action along this trajectory. The Van-Vleck prefactor $C_{V V}\left(\mathbf{q}_{0}, \mathbf{p}_{0} ; t\right)$ and the Herman-Kluk prefactor $C_{H K}\left(\mathbf{q}_{0}, \mathbf{p}_{0} ; t\right)$ involve elements of the stability matrix ${ }^{25}$

$$
\begin{gathered}
C_{V V}\left(\mathbf{q}_{0}, \mathbf{p}_{0} ; t\right)=\left\{\operatorname{det}\left[\mathbf{M}_{q p}\right] /(2 \pi i \hbar)^{f}\right\}^{1 / 2} \\
C_{H K}\left(\mathbf{q}_{0}, \mathbf{p}_{0} ; t\right)=\left\{\operatorname{det}\left[\frac{1}{2}\left(\mathbf{M}_{q q}+\mathbf{M}_{p p}+\frac{\hbar \gamma}{i} \mathbf{M}_{q p}+\frac{i}{\hbar \gamma} \mathbf{M}_{p q}\right)\right]\right\}^{1 / 2}
\end{gathered}
$$

where

$$
\begin{aligned}
& \mathbf{M}_{q q}=\frac{\partial \mathbf{q}_{t}}{\partial \mathbf{q}_{0}}, \quad \mathbf{M}_{q p}=\frac{\partial \mathbf{q}_{t}}{\partial \mathbf{p}_{0}} \\
& \mathbf{M}_{p q}=\frac{\partial \mathbf{p}_{t}}{\partial \mathbf{q}_{0}}, \quad \mathbf{M}_{p p}=\frac{\partial \mathbf{p}_{t}}{\partial \mathbf{p}_{0}}
\end{aligned}
$$

The wavefunction for the coherent $\operatorname{states}^{26}$ in Eq. (6) is

$$
\langle\mathbf{x} \mid \mathbf{q p}\rangle=\left(\frac{\gamma}{\pi}\right)^{f / 4} \exp \left[-\frac{\gamma}{2}(\mathbf{x}-\mathbf{q})^{2}+\frac{i}{\hbar} \mathbf{p} \cdot(\mathbf{x}-\mathbf{q})\right]
$$

In the limit that the coherent state parameter $\gamma$ goes to $\infty$, the Herman-Kluk propagator of Eq. (6) reverts back to the Van-Vleck propagator of Eq. (5). It has been shown that the Herman-Kluk propagator can also be derived by inserting the Van-Vleck propagator into the time evolved wave function expression and applying a modified Filinov filtering to it. ${ }^{21,24}$ Inserting the HK-IVR expression for the two time evolution operators in the flux-correlation function of Eq. (2), one gets

$$
\begin{aligned}
C_{f f}(t)= & (2 \pi \hbar)^{-2 f} \int d \mathbf{q}_{0} \int d \mathbf{p}_{0} \int d \mathbf{q}_{0}^{\prime} \int d \mathbf{p}_{0}^{\prime} \\
& \left\langle\mathbf{q}_{0} \mathbf{p}_{0}|\hat{\mathcal{F}}(\beta)| \mathbf{q}_{0}^{\prime} \mathbf{p}_{0}^{\prime}\right\rangle\left\langle\mathbf{q}_{t}^{\prime} \mathbf{p}_{t}^{\prime}|\hat{F}| \mathbf{q}_{t} \mathbf{p}_{t}\right\rangle \\
& C_{H K}\left(\mathbf{q}_{0}, \mathbf{p}_{0} ; t\right) C_{H K}^{*}\left(\mathbf{q}_{0}^{\prime}, \mathbf{p}_{0}^{\prime} ; t\right) e^{i\left[S_{t}\left(\mathbf{q}_{0} \mathbf{p}_{0}\right)-S_{t}\left(\mathbf{q}_{0}^{\prime} \mathbf{p}_{0}^{\prime}\right)\right] / \hbar} .
\end{aligned}
$$

This gives rise to a double phase space average ${ }^{31}$ where one runs a pair of trajectories with initial conditions $\left(\mathbf{q}_{0}, \mathbf{p}_{0}\right)$ and $\left(\mathbf{q}_{0}^{\prime}, \mathbf{p}_{0}^{\prime}\right)$ for time $t$. The integrand of this expression is highly oscillatory and it can be evaluated only for systems with a few degrees of freedom. Hence T.Yamamoto and Miller employed a more symmetrized version of the flux correlation function; this considerably reduced the oscillatory nature of the double phase space average. This methodology was successfully tested on various problems; this includes the Eckart barrier, system-bath models with more than 100 dof and a benchmark gas phase reaction $D+$ 
$\mathrm{H}_{2}{ }^{32}$ However the plane wave terms in the coherent state matrix elements posed considerable challenges and it was difficult to apply this to further higher dimensional systems. Since the evaluation of the coherent state matrix element of the Boltzmannized flux operator is more demanding than the corresponding coordinate matrix element, it is thus desirable to use the coordinate state Van-Vleck propagator instead of the coherent state Herman-Kluk. Inserting the VV-IVR expression for the two time evolution operators in Eq. (2) one gets

$$
\begin{aligned}
C_{f f}(t)= & \int d \mathbf{q}_{0} \int d \mathbf{p}_{0} \int d \mathbf{q}_{0}^{\prime} \int d \mathbf{p}_{0}^{\prime}\left\langle\mathbf{q}_{0}|\hat{\mathcal{F}}(\beta)| \mathbf{q}_{0}^{\prime}\right\rangle\left\langle\mathbf{q}_{t}^{\prime}|\hat{F}| \mathbf{q}_{t}\right\rangle \\
& C_{V V}\left(\mathbf{q}_{0}, \mathbf{p}_{0} ; t\right) C_{V V}\left(\mathbf{q}_{0}^{\prime}, \mathbf{p}_{0}^{\prime} ; t\right) e^{i\left[S_{t}\left(\mathbf{q}_{0} \mathbf{p}_{0}\right)-S_{t}\left(\mathbf{q}_{0}^{\prime} \mathbf{p}_{0}^{\prime}\right)\right] / \hbar} .
\end{aligned}
$$

This expression has the same double phase structure as Eq. (11). The flux operator however forces the two trajectories to end at the same point on the dividing surface i.e $\mathbf{q}_{t}=\mathbf{q}_{t}^{\prime}$ and this should lie on the dividing surface $s(\mathbf{q})=0$. This suggests that one can get a much simpler expression if we start the two trajectories on the dividing surface and evolve them negatively in time. The two time evolution operators are rewritten as :

$$
\begin{aligned}
e^{i \hat{H} t / \hbar} & =\int d \mathbf{q}_{0}^{\prime} \int d \mathbf{p}_{0}^{\prime} C_{V V}\left(\mathbf{q}_{0}^{\prime}, \mathbf{p}_{0}^{\prime} ;-t\right) e^{i S_{-t}\left(\mathbf{q}_{0}^{\prime}, \mathbf{p}_{0}^{\prime}\right) / \hbar}\left|\mathbf{q}_{-t}^{\prime}\right\rangle\left\langle\mathbf{q}_{0}^{\prime}\right| \\
e^{-i \hat{H} t / \hbar} & =\int d \mathbf{q}_{0} \int d \mathbf{p}_{0} C_{V V}^{*}\left(\mathbf{q}_{0}, \mathbf{p}_{0} ;-t\right) e^{-i S_{-t}\left(\mathbf{q}_{0}, \mathbf{p}_{0}\right) / \hbar}\left|\mathbf{q}_{0}\right\rangle\left\langle\mathbf{q}_{-t}\right| .
\end{aligned}
$$

Inserting these two time propagators into the flux correlation function gives

$$
\begin{gathered}
C_{f f}^{V V-I V R}(t)=\int d \mathbf{q}_{0} \int d \mathbf{p}_{0} \int d \mathbf{p}_{0}^{\prime} C_{V V}^{*}\left(\mathbf{q}_{0}, \mathbf{p}_{0} ;-t\right) C_{V V}\left(\mathbf{q}_{0}, \mathbf{p}_{0}^{\prime} ;-t\right) \\
\left\langle\mathbf{q}_{-t}|\hat{\mathcal{F}}(\beta)| \mathbf{q}_{-t}^{\prime}\right\rangle\left\langle\mathbf{q}_{0}^{\prime}|\hat{F}| \mathbf{q}_{0}\right\rangle \\
e^{i S_{-t}\left(\mathbf{q}_{0}, \mathbf{p}_{0}^{\prime}\right) / \hbar} e^{-i S_{-t}\left(\mathbf{q}_{0}, \mathbf{p}_{0}\right) / \hbar}
\end{gathered}
$$

The coordinate matrix element of the flux operator is given by:

$$
\left\langle\mathbf{q}_{0}^{\prime}|\hat{F}| \mathbf{q}_{0}\right\rangle=\frac{\hbar}{2 i m}\left\{\delta\left(s\left(\mathbf{q}_{0}\right)\right) \nabla s\left(\mathbf{q}_{0}\right) \cdot \frac{\partial \delta\left(\mathbf{q}_{0}^{\prime}-\mathbf{q}_{0}\right)}{\partial \mathbf{q}_{0}^{\prime}}-\delta\left(s\left(\mathbf{q}_{0}^{\prime}\right)\right) \nabla s\left(\mathbf{q}_{0}^{\prime}\right) \cdot \frac{\partial \delta\left(\mathbf{q}_{0}^{\prime}-\mathbf{q}_{0}\right)}{\partial \mathbf{q}_{0}}\right\}
$$

The flux operator localizes the initial positions $\mathbf{q}_{0}$ and $\mathbf{q}_{0}^{\prime}$ to lie on the same point on the dividing surface. The Van-Vleck IVR flux correlation function becomes

$$
\begin{gathered}
C_{f f}^{V V-I V R}(t)=\int d \mathbf{q}_{0} \int d \mathbf{p}_{0} \int d \mathbf{p}_{0}^{\prime} C_{V V}^{*}\left(\mathbf{q}_{0}, \mathbf{p}_{0} ;-t\right) C_{V V}\left(\mathbf{q}_{0}, \mathbf{p}_{0}^{\prime} ;-t\right)\left\langle\mathbf{q}_{-t}|\hat{\mathcal{F}}(\beta)| \mathbf{q}_{-t}^{\prime}\right\rangle \\
\frac{\left(\mathbf{p}_{0}+\mathbf{p}_{0}^{\prime}\right)}{2 m} \cdot \nabla s\left(\mathbf{q}_{0}\right) \delta\left(s\left(\mathbf{q}_{0}\right)\right) \\
e^{i S_{-t}\left(\mathbf{q}_{0}, \mathbf{p}_{0}^{\prime}\right) / \hbar} e^{-i S_{-t}\left(\mathbf{q}_{0}, \mathbf{p}_{0}\right) / \hbar}
\end{gathered}
$$

One thus evolves two classical trajectories for time $-t$ starting at the same initial position $\mathbf{q}_{0}$ which lies on the dividing surface $s\left(\mathbf{q}_{0}\right)=0$ with different initial momenta $\mathbf{p}_{0}$ and $\mathbf{p}_{0}^{\prime}$. This expression has no further approximation other than the semiclassical IVR for the two 
time propagators and it is much simpler than the double phase expression of Herman-Kluk since it has only three integration variables. Also the integrand is less oscillatory because it has the coordinate elements of the Boltzmannized flux operator. Since the trajectories start at the same initial point $\mathbf{q}_{0}$ and runs with different initial momenta $\mathbf{p}_{0}$ and $\mathbf{p}_{0}^{\prime}$ the action of the two trajectories partially cancel each other.

One can also get an alternative linearized IVR expression for the flux correlation function if we assume that the two trajectories are near each other. We then change integration variables from $\mathbf{p}_{0}$ and $\mathbf{p}_{0}^{\prime}$ to $\overline{\mathbf{p}}_{0}=\frac{\mathbf{p}_{0}+\mathbf{p}_{0}^{\prime}}{2}$ and $\Delta \mathbf{p}_{0}=\mathbf{p}_{0}-\mathbf{p}_{0}^{\prime}$ and make a linear expansion of all quantities in the variable $\Delta \mathbf{p}_{0}$. The linearized IVR flux correlation function then becomes

$$
C_{f f}^{L S C-I V R}(t)=\int d \overline{\mathbf{q}}_{0} \int d \overline{\mathbf{p}}_{0}(\hat{\mathcal{F}}(\beta))_{W}\left(\overline{\mathbf{q}}_{-t}, \overline{\mathbf{p}}_{-t}\right)(\hat{F})_{W}\left(\overline{\mathbf{q}}_{0}, \overline{\mathbf{p}}_{0}\right)
$$

where $A_{W}(\mathbf{q}, \mathbf{p})$ is the Wigner transform of the operator $\hat{A}$ and it is given by :

$$
A_{W}(\mathbf{q}, \mathbf{p})=\int d \Delta \mathbf{q} e^{-i \mathbf{p}^{T} \cdot \Delta \mathbf{q} / \hbar}\left\langle\mathbf{q}+\frac{\Delta \mathbf{q}}{2}|\hat{A}| \mathbf{q}-\frac{\Delta \mathbf{q}}{2}\right\rangle
$$

Here, one starts a single trajectory with the initial position $\overline{\mathbf{q}}_{0}$ on the dividing surface and initial momentum $\overline{\mathbf{p}}_{0}$ and evolves it for negative time $-t$. One notices that the Van-Vleck IVR expression of Eq. (17) has the same computational complexity as the Linearized IVR.

The only awkward feature of this formulation is that the Boltzmannized Flux operator $\hat{\mathcal{F}}(\beta)$ localizes the final positions $\mathbf{q}_{-t}$ and $\mathbf{q}_{-t}^{\prime}$. The trajectories at time $-t$ have to land in the transition state region. So the region of initial phase space that one ends up sampling will vary with time. For the simple few degree of freedom systems that we consider in this paper, one can use grid based methods or un-weighted Monte-Carlo procedure to calculate the SC-IVR time correlation functions. We also used the recently proposed time dependent sampling ${ }^{38}$ procedure to sample the initial phase space. The natural weight function is $\rho\left(\mathbf{q}_{0}, \mathbf{p}_{0}, \mathbf{p}_{0}^{\prime} ; t\right)=\left|\left\langle\mathbf{q}_{-t}|\hat{\mathcal{F}}(\beta)| \mathbf{q}_{-t}^{\prime}\right\rangle\right|$, and one then uses the standard Metropolis ${ }^{37}$ criteria to accept or reject a configuration. The time dependent normalization constant would then be calculated using log-derivative formalism as outlined in that paper.

\section{$3 \quad$ Model problems}

\subsection{Eckart Barrier}

The first example we consider is transmission through a one dimensional Eckart barrier

$$
V(q)=\frac{V_{0}}{\cosh ^{2}(a q)},
$$

The parameters correspond approximately to the $\mathrm{H}+\mathrm{H}_{2}$ reaction: $m=1061.0$ a.u., $V_{0}=$ $0.425 \mathrm{eV}$, and $a=1.36 \mathrm{a} . \mathrm{u}$. For this $1-\mathrm{D}$ model problem, the initial position $q_{0}$ is located on top of the barrier. This gets rid of the position integral and one is left with the 2 dimensional integral over initial momenta. Thus the real time trajectories that contribute to the rate are the trajectories with energy greater than or equal to the barrier height $V_{0}$. The matrix 
elements of the Boltzmannized flux operator are calculated quantum mechanically. The exact quantum mechanical results are obtained using the discrete variable representations (DVR). ${ }^{34}$ Figure 1 shows the flux-flux correlation function at several temperatures obtained via the above methodology (labeled as VV-IVR), the exact quantum mechanical result and via the linearized SC-IVR. Here the flux correlation function shows the typical behavior of a "direct" reaction, and decays to zero in time $\hbar \beta$. At high temperature the Van-Vleck flux correlation function exactly matches the quantum correlation function; however they start deviating in the deep tunneling regime.

Arrhenius plots of the thermal rate constants are shown in Figure 2 and the percentage error in Figure 3. The VV-IVR method provides an excellent agreement with the exact quantum result from $T=2000 \mathrm{~K}$ to $T=150 \mathrm{~K}$. The difference between the Van-Vleck and the quantum rates are within $4 \%$ error for the temperature of $300-2000 \mathrm{~K}$ and around 33.5 $\%$ at the lowest temperature $T=150 \mathrm{~K}$. The LSC-IVR rates are within $10 \%$ error for the temperature range $300 \mathrm{~K}-2000 \mathrm{~K}$ and $35 \%$ error at $T=200 \mathrm{~K}$. As has been documented earlier the LSC-IVR ${ }^{28,31}$ seems to miss some quantum effects in its real time propagation.

\subsection{Double well}

The second example we consider is a 1-D double well potential

$$
V(s)=-\frac{1}{2} m_{s} w_{b}^{2} s^{2}+\frac{m_{s}^{2} w_{b}^{4}}{16 V_{o}} s^{4}
$$

The mass of the particle is $m_{s}=1836.1 a . u$., the barrier height is $V_{o}=2085 \mathrm{~cm}^{-1}$ and the barrier frequency is $w_{b}=500 \mathrm{~cm}^{-1}$. As in the previous example, the dividing surface is chosen to be on top of the barrier. This example provides a stringent test of the ability of our formulation to describe quantum coherence/interference effects. Unlike the previous example, the flux correlation function does not decay to zero and the particle oscillates back and forth in the double well. The recrossing dynamics persists forever and the rate constant $k(T)$ does not exist. Figure 4 shows the full VV-IVR, linearized SC-IVR and the exact quantum mechanical results for times well into the recrossing regime at temperature $T=900 K$ and $300 K$. The correlation function shows a smooth decay upto around $25 \mathrm{fs}$ for $\mathrm{T}=900 \mathrm{~K}$. The Van-Vleck IVR and the LSC-IVR correlation functions accurately capture this regime. After $25 \mathrm{fs}$ one notices that there is a lot of recrossing dynamics. Even though the LSC-IVR seems to capture some of the recrossing dynamics till $t=70 \mathrm{fs}$, it starts deviating very drastically at longer times. The quantum coherence feature which arises from the interference of two distinct classical trajectories cannot be described by this approximation. However the full VV-IVR has the ability to describe this regime. Similarly at $\mathrm{T}=300 \mathrm{~K}$ the smooth exponential regime is till $t=50 \mathrm{fs}$ for which both the VV-IVR and LSC-IVR results agrees with the quantum result. The VV-IVR is able to describe the recrossing dynamics present beyond $t>50 \mathrm{fs}$ accurately, though it has a slight phase lag. Even though the effort required to do the VV-IVR calculation is the same as the Linearized IVR, we were able to propagate the LSC-IVR for longer times (greater than $250 \mathrm{fs}$ ) than the corresponding full VV-IVR. This is because the VV-IVR integrand is certainly more oscillatory than the approximate Linearized IVR. A Filinov smoothing of the VV-IVR integrand might help in alleviating the wild oscillations. 


\subsection{Collinear $\mathrm{H}+\mathrm{H}_{2}$}

Another example we consider is that of the collinear Hydrogen exchange reaction $\mathrm{H}+\mathrm{H}_{2} \rightarrow$ $H_{2}+H$ using the LSTH potential energy surface. ${ }^{35}$ The calculations were performed using the mass scaled normal mode coordinates $(s, Q)$ about the transition state. Here $s$ is the reaction coordinate and $Q$ the bound coordinate. The choice of the dividing surface is very similar to the earlier case and it is at $s=0$. It has been observed earlier that the constraint of collinearity leads to recrossing effects in this model. ${ }^{36}$ Figure 5 shows the plot of the flux correlation function at $T=2400 \mathrm{~K}, 1000 \mathrm{~K}$ and $400 \mathrm{~K}$; the Arrhenius rates appear in Figure 6. At high temperature, the exact $C_{f f}(t)$ shows a lot of recrossing and the SC-IVR is able to capture it accurately. At low temperature the recrossing is totally quenched. The VV-IVR calculation gets progressively difficult as we go down to lower temperature. For the temperature range of $400 \mathrm{~K}$ to $2400 \mathrm{~K}$ the VV-IVR rates are within $15 \%$ error of the exact quantum result. The LSC-IVR rate however reaches a $50 \%$ error at the lowest temperature.

\section{Conclusion}

In section 2 we have shown how the SC-IVR in the Van-Vleck formulation can be used to calculate flux correlation functions. In this model, one starts trajectories from the dividing surface that separates reactants and products and then evolves it for (negative) time $-t$. The resulting final positions are required to be in the transition state region. The advantage of this formulation is that it has the same computational effort as the classical Wigner model. The results for the various model problems are quite encouraging.

Like other IVR formulations one still has to deal with an oscillatory integrand; this has limited us in practice to times upto only a few hundred femtoseconds. The SC-IVR calculation was also quite difficult to converge at low temperature for the Collinear $\mathrm{H}+\mathrm{H}_{2}$. This could be improved if we combine this formulation with the well known Stationary phase filtering techniques.

\section{Acknowledgments}

This work was supported by the Office of Naval Research Grant No. N00014-05-1-0457 and by the Director, Office of Science, Office of Basic Energy Sciences, Chemical Sciences, Geosciences, and Biosciences Division, U.S. Department of Energy under Contract No. DEAC02-05CH11231.

\section{References}

[1] R. Kubo, J. Phys. Soc. Japan. 12, 570, (1957).

[2] R. Zwanzig, Ann. Rev. Phys. Chem. 16, 67 (1965).

[3] W. H. Miller, J. Chem. Phys. 61, 1823 (1974); W. H. Miller, S. D. Schwartz and J. W. Tromp, J. Chem. Phys. 79, 4889 (1983). 
[4] T. Yamamoto, J. Chem. Phys. 33, 281 (1960).

[5] J. Z. H. Zhang, Theory and Application of Quantum Molecular Dynamics, (World Scientific, Singapore, 1999).

[6] R. P. Feynman and A. R. Hibbs, Quantum Mechanics and Path Integrals, (McGraw-Hill, New York, 1965).

[7] B. J. Berne and D. Thirumalai, Ann. Rev. Phys. Chem. 37, 401 (1986); C. H. Mak and D. Chandler, Phys. Rev. A 41, 5709 (1990); J. D. Doll, D. L. Freeman and T. L. Beck, Adv. Chem. Phys. 78, 61 (1994);C. H. Mak, R. Egger and H. Weber-Gottschick, Phys. Rev. Lett. 81, 4533 (1998).

[8] K. Yamashita and W. H. Miller, J. Chem. Phys. 82, 5475 (1985); J. W. Tromp and W. H. Miller, J. Phys. Chem. 90, 3482 (1986).

[9] G. Krilov, E. Sim and B. J. Berne, J. Chem. Phys. 114, 1075 (2001); E. Rabani, G. Krilov and B. J. Berne, Chem.Phys. 112, 2605 (2000); E. Sim, G. Krilov and B. J. Berne, J. Phys. Chem. A, 105, 2824 (2001); E. Rabani, D. R. Reichman and G. Krilov and B. J. Berne, Proc. Nat. Acad. Sci. USA. 99, 1129 (2002).

[10] S. Hammes-Schiffer and J. C. Tully, J. Chem. Phys. 103, 8528 (1995); D. F. Coker, H. S. Mei and J. P. Ryckaert, in Classical and Quantum Dynamics in Condensed Phase Simulations, edited by B. J. Berne, G. Ciccotti and D. F. Coker, (World Scientific, Singapore, 1998).

[11] S. A. Egorov, E. Rabani and B.J. Berne, J. Chem. Phys. 110, 5238 (1999); S. A. Egorov, Eran Rabani and B. J. Berne, J. Phys. Chem. B 103, 10978 (1999).

[12] E. Wigner, Trans. Faraday. Soc. 34, 29 (1938).

[13] H. Eyring, Trans. Faraday. Soc. 34, 41 (1938).

[14] W. H. Miller, J. Chem. Phys. 62, 1899 (1975). G. A. Voth, D. Chandler and W. H. Miller, J. Chem. Phys. 91, 7749 (1989); D. G. Truhlar and B. C. Garrett, J. Phys. Chem. 96, 6515 (1992); N. F. Hansen and H. C. Andersen, J. Phys. Chem. 100, 1137 (1996).

[15] W. H. Miller, Y. Zhao, M. Ceotto and S. Yang, J. Chem. Phys. 119, 1329 (2003); C. Venkataraman and W. H. Miller, J. Phys. Chem. A 108, 3035 (2004); M. Ceotto and W. H. Miller, J. Chem. Phys. 120, 6356 (2004); T. Yamamoto and W. H. Miller, J. Chem. Phys. 120, 3086 (2004); Y. Zhao, T. Yamamoto and W. H. Miller, J. Chem. Phys. 120, 3100 (2004); T. Yamamoto and W. H. Miller, J. Chem. Phys. 122, 044106 (2005); J. Vanicek, W. H. Miller, J. F. Castillo and F. J. Aoiz, J. Chem. Phys. 123, 054108 (2005).

[16] K. G. Kay, J. Chem. Phys. 100, 4377 (1994); K. G. Kay, J. Chem. Phys. 100, 4432 (1994). 
[17] W. H. Miller, Adv. Chem. Phys. 25, 69 (1974); 30, 77 (1975).

[18] W. H. Miller, Faraday. Discuss. 110, 1 (1998); J. Phys. Chem. A 105, 2942 (2001).

[19] J. V. Van Vleck, Proc. Nat. Acad. Sci. USA. 14, 178 (1928).

[20] M. F. Herman and E. Kluk, Chem. Phys. 91, 27 (1984); E. Kluk, M. F. Herman, and H.L. Davis, J. Chem. Phys. 84, 326 (1986).

[21] W. H. Miller, Mol. Phys. 100, 397 (2002).

[22] M. Baranger, M. A. M. de Aguiar, F. Keck, H. J. Korsch, and B. Schellhaas, J. Phys. A: Math. Gen. 34, 7227 (2001).

[23] E. J. Heller, J. Chem. Phys. 94, 2723 (1991); W. H. Miller, J. Chem. Phys. 53, 3578 (1970); 95, 9428 (1991);E. J. Heller, J. Chem. Phys. 95, 9431 (1991)

[24] V. S. Filinov, Nuc. Phys. B 271, 717 (1986); N. Makri and W. H. Miller, Chem. Phys. Lett. 139, 10 (1987); J. Chem. Phys. 89, 2170 (1988); J. D. Doll and D. L. Freeman, Adv. Chem. Phys. 73, 289 (1989).

[25] H. Goldstein, Classical Mechanics, 2nd ed. (Addison-Wesley, New York, 1988).

[26] R. J. Glauber, Phys. Rev. 130, 2529 (1963); 131, 2766 (1963); J. R. Klauder and B. S. Skagerstam, Coherent States (World Scientific, River Edge, NJ, 1985).

[27] H. B. Wang, X. Sun and W. H. Miller, J. Chem. Phys. 108, 9726 (1998).

[28] X. Sun and W. H. Miller, J. Chem. Phys. 106, 916 (1997); X. Sun, H. B. Wang and W. H. Miller, J. Chem. Phys. 109, 4190 (1998); X. Sun, H. B. Wang and W. H. Miller, J. Chem. Phys. 109, 7064 (1998); W. H. Miller, J. Phys. Chem. A 103, 9384 (1999).

[29] X. Sun and W. H. Miller, J. Chem. Phys. 110, 6635 (1999).

[30] M. Thoss, H. Wang and W. H. Miller, J. Chem. Phys. 114, 9220 (2001).

[31] T. Yamamoto, H. Wang and W. H. Miller, J. Chem. Phys. 116, 7335 (2002).

[32] T. Yamamoto and W. H. Miller, J. Chem. Phys. 118, 2135 (2003).

[33] N. Makri and K. Thompson, Chem. Phys. Lett. 291, 101 (1998); N. Makri and K. Thompson, J. Chem. Phys. 110, 1343 (1999).

[34] J. C. Light, I. P. Hamilton and J. V. Lill, J. Chem. Phys. 82, 1400 (1985); D. T. Colbert and W. H. Miller, J. Chem. Phys. 96, 1982 (1992); G. C. Groenenboom and D.T. Colbert, J. Chem. Phys. 99, 9681 (1993).

[35] P. Siegbahn and B. Liu, J. Chem. Phys. 68, 2457 (1978); D. G. Truhlar and C. J. Horowitz, J. Chem. Phys. 68, 2466 (1978).

[36] S. Chapman, S. M. Hornstein, and W. H. Miller J. Am. Chem. Soc. 97, 892 (1975). 
[37] N. Metropolis, A. W. Rosenbluth, M. N. Rosenbluth, A. H. Teller, and E. Teller, J. Chem. Phys. 21, 1087 (1953).

[38] Y. Li, N. Ananth and W. H. Miller (to be published). 
Figure 1: The flux-flux correlation function $C_{f f}(t)$ for the one-dimensional Eckart barrier at $T=1000 \mathrm{~K}$ [panel (a)], $T=300 \mathrm{~K}[\operatorname{panel}(\mathrm{b})$ and $T=200 \mathrm{~K}[$ panel(c)]. The solid, dashdotted, and the dashed curves correspond to the results of the exact quantum mechanical calculation, the present method (VV-IVR) and the linearized IVR.

Figure 2: An Arrhenius plot of the thermal rate constant for the one-dimensional Eckart barrier. The dashed and solid lines show the classical and exact quantum mechanical rates, respectively, while circles and squares correspond to the result of the Van-Vleck IVR and the linearized IVR, respectively.

Figure 3: Percentage error plot of the thermal rate constant for the one-dimensional Eckart barrier. The circles and squares correspond to the result of the Van-Vleck IVR and the Linearized IVR, respectively.

Figure 4: The flux-flux correlation function $C_{f f}(t)$ for the one-dimensional double well at $T=900 \mathrm{~K}$ [panel (a)], $T=300 \mathrm{~K}$ [panel(b)]. The solid line, filled-circles, and dashed line correspond to the results of the exact quantum mechanical calculation, the Van-Vleck IVR and the linearized IVR.

Figure 5: The flux-flux correlation function $C_{f f}(t)$ for the collinear $H+H_{2}$ at $T=2400 \mathrm{~K}$ [panel (a)], $T=1000 \mathrm{~K}[\operatorname{panel}(\mathrm{b})$ and $T=400 \mathrm{~K}[\operatorname{panel}(\mathrm{c})]$. The solid, dash-dotted, and dashed curves correspond to the results of the exact quantum mechanical calculation, the present method (VV-IVR) and the linearized SC-IVR.

Figure 6: A plot of the thermal rate constant for the collinear $\mathrm{H}+\mathrm{H}_{2}$. The circles show the exact quantum mechanical rates, while the squares and triangle correspond to the result of the Van-Vleck IVR and the Linearized IVR, respectively. 


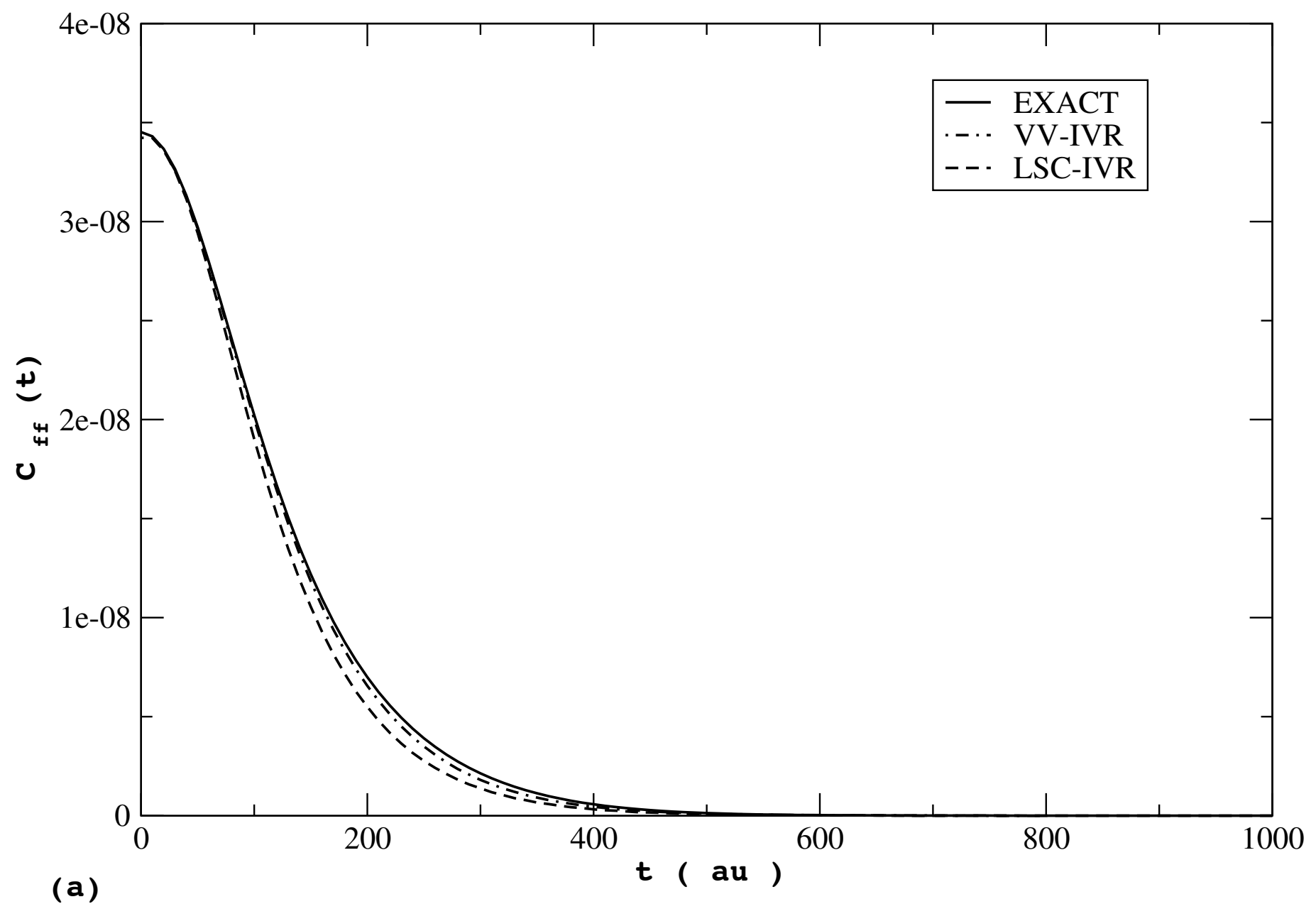




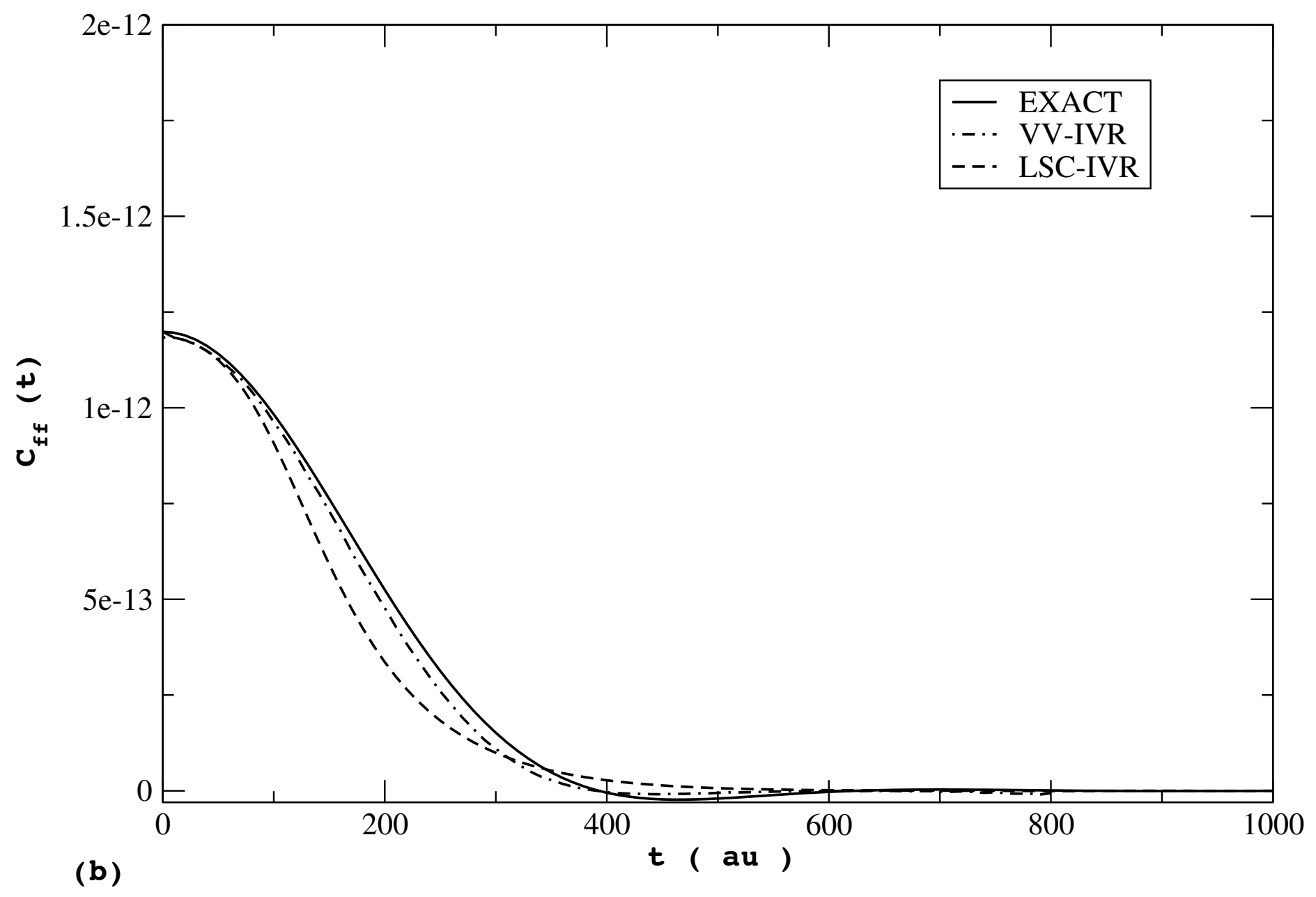




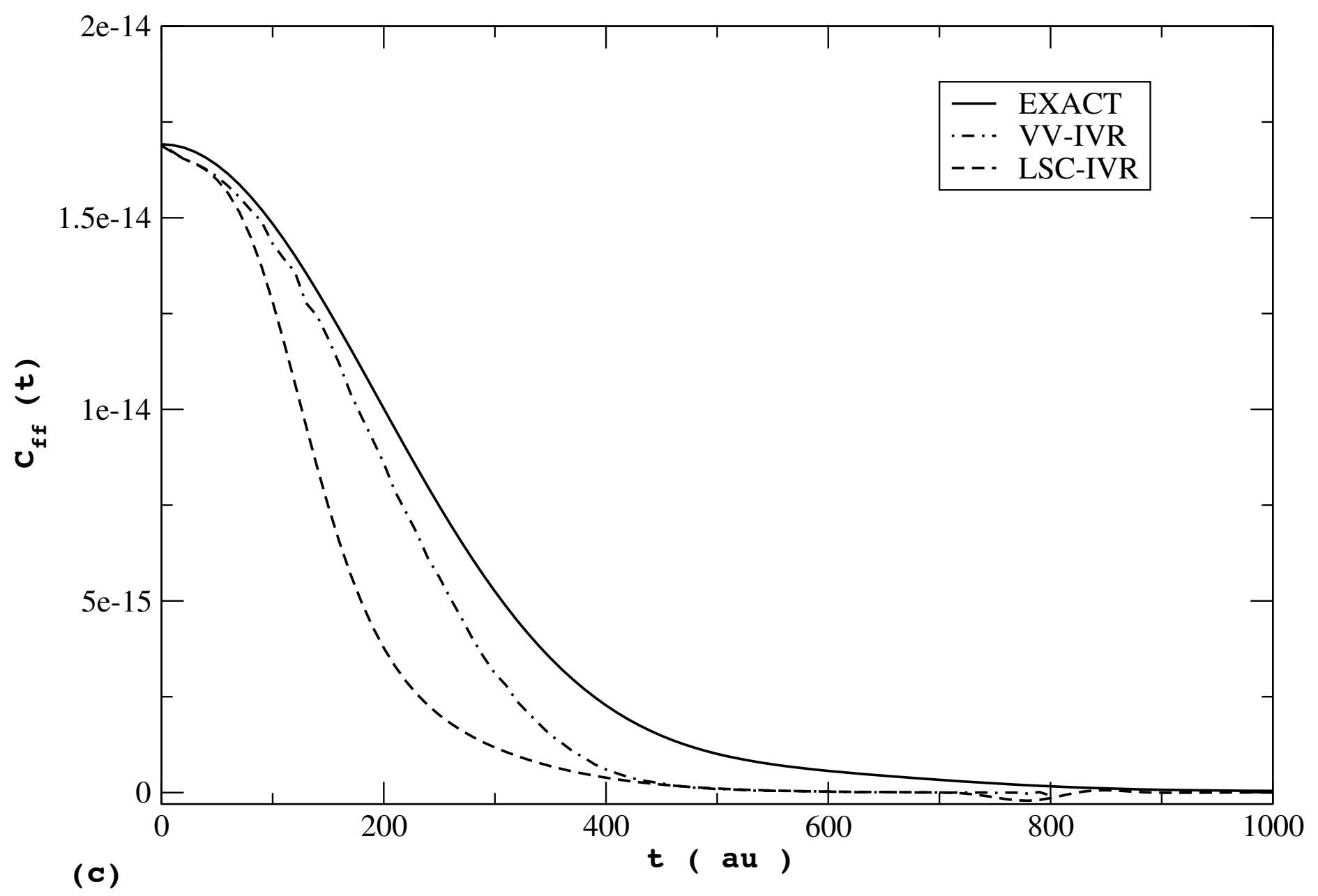




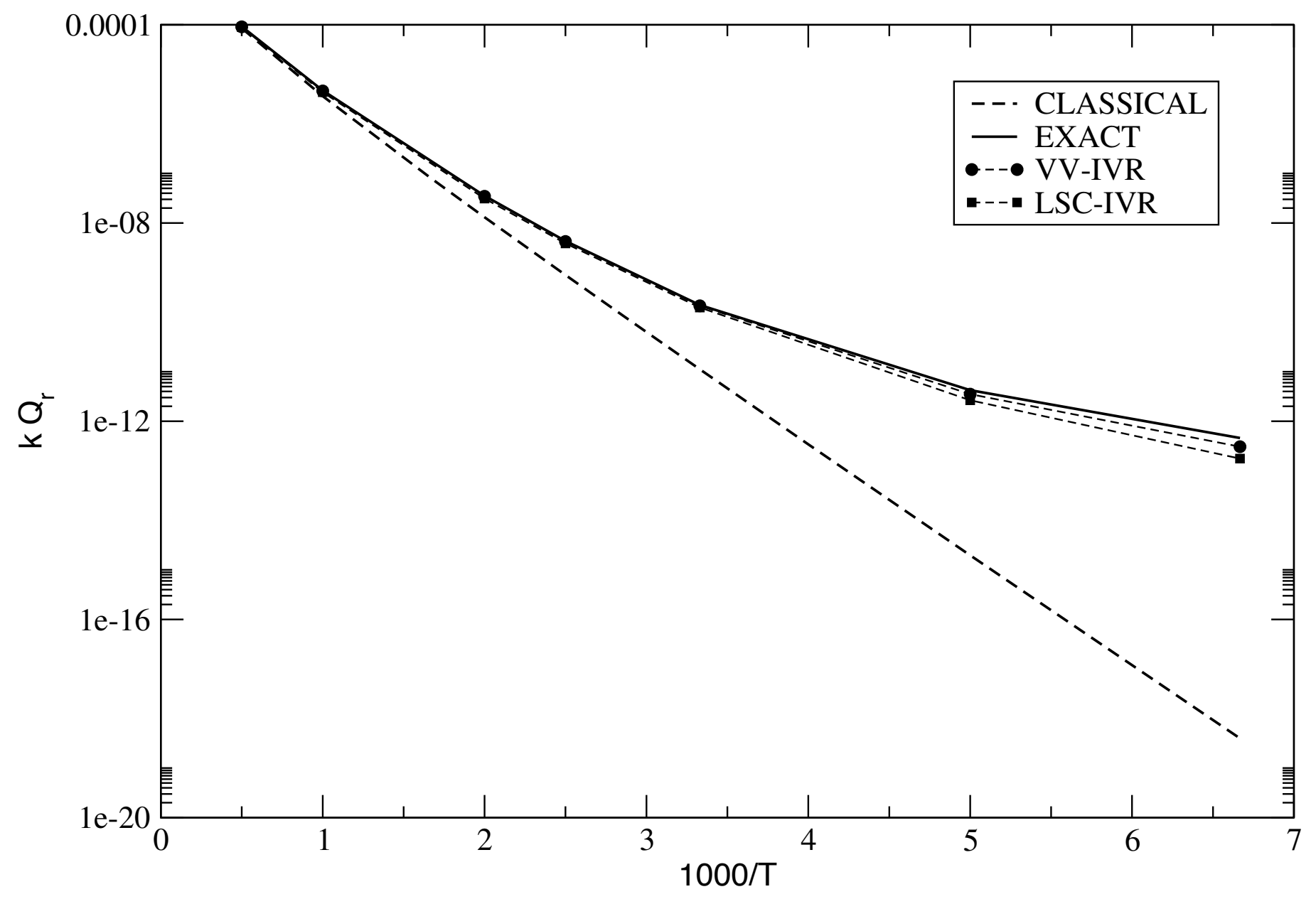




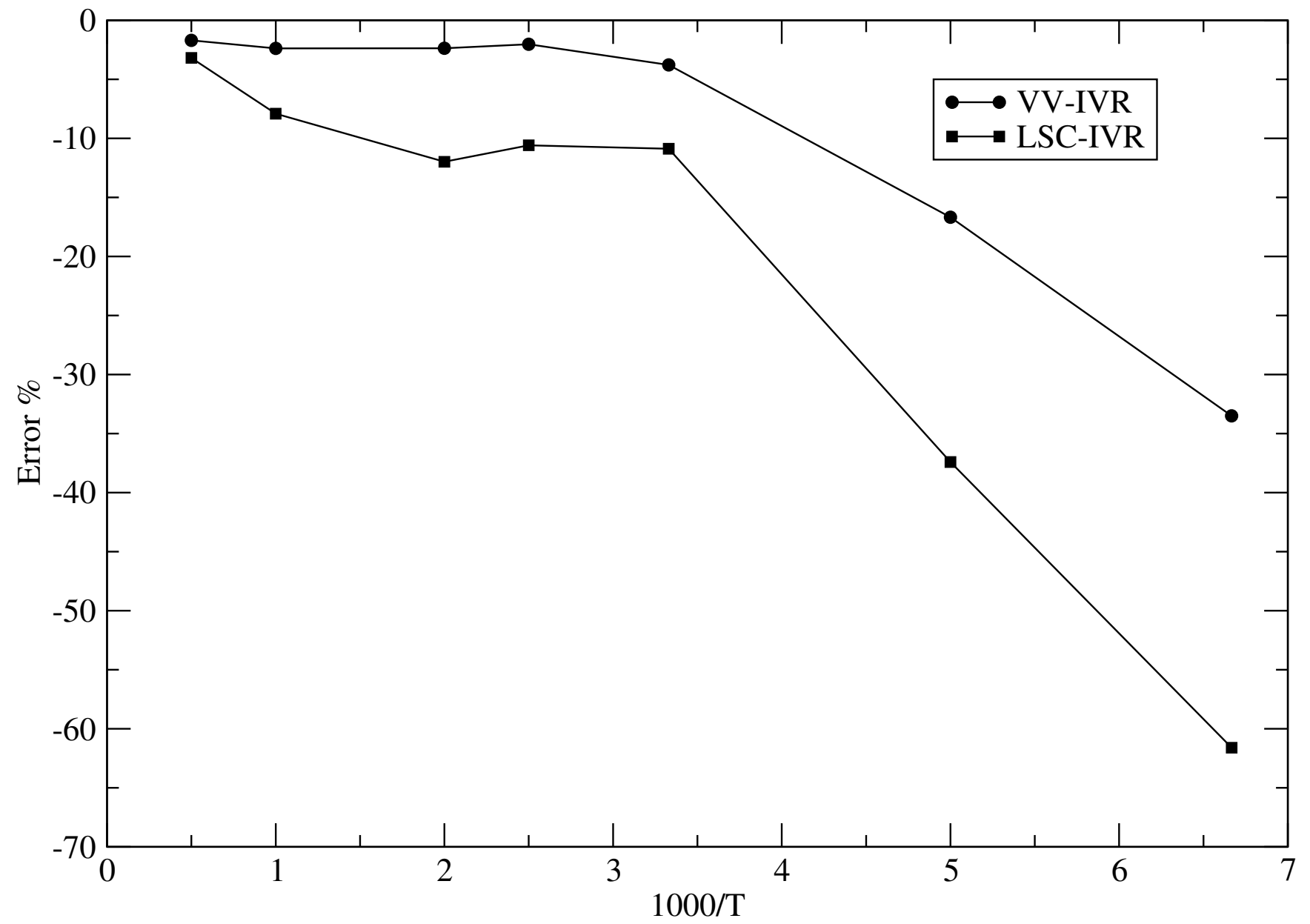




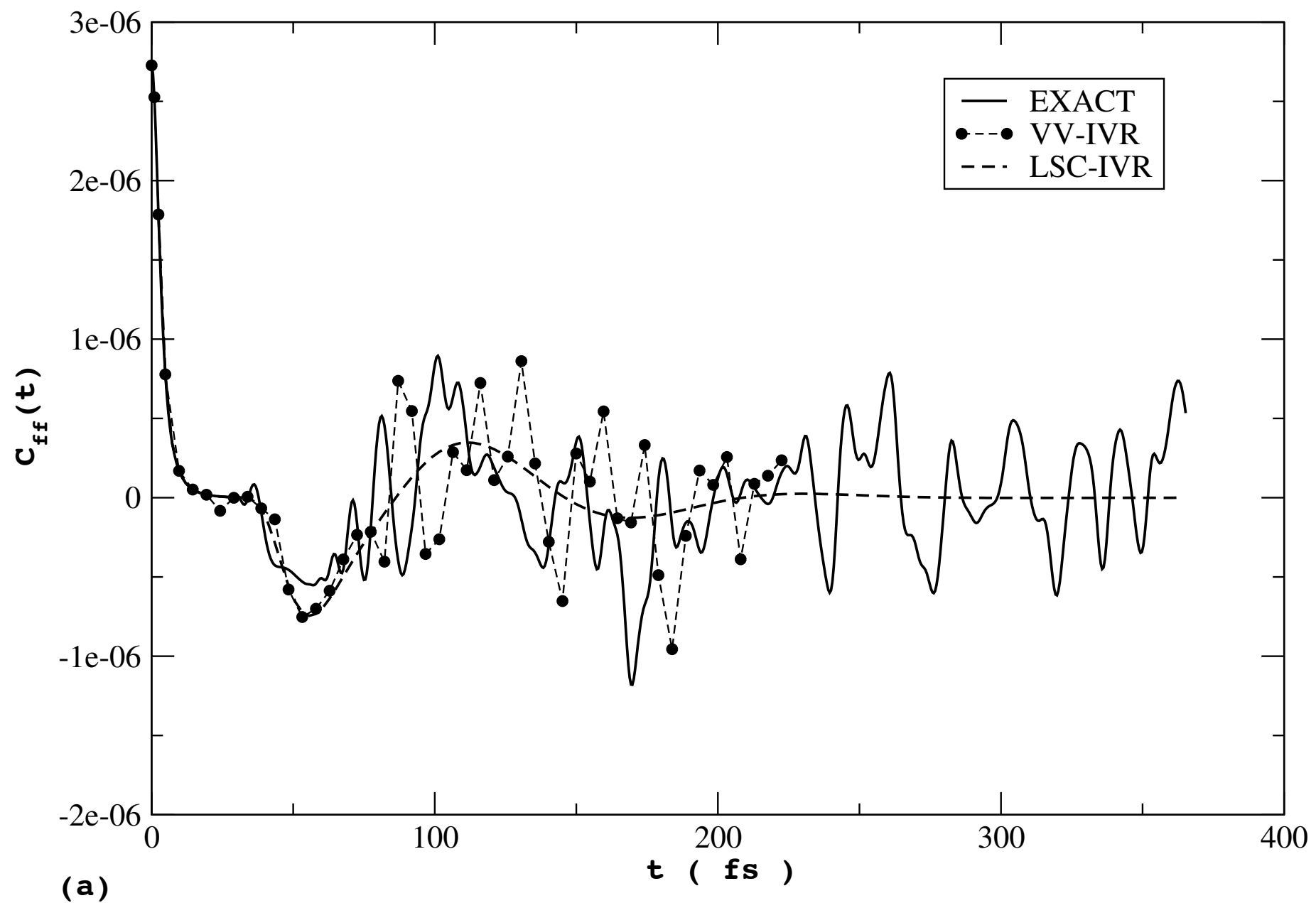




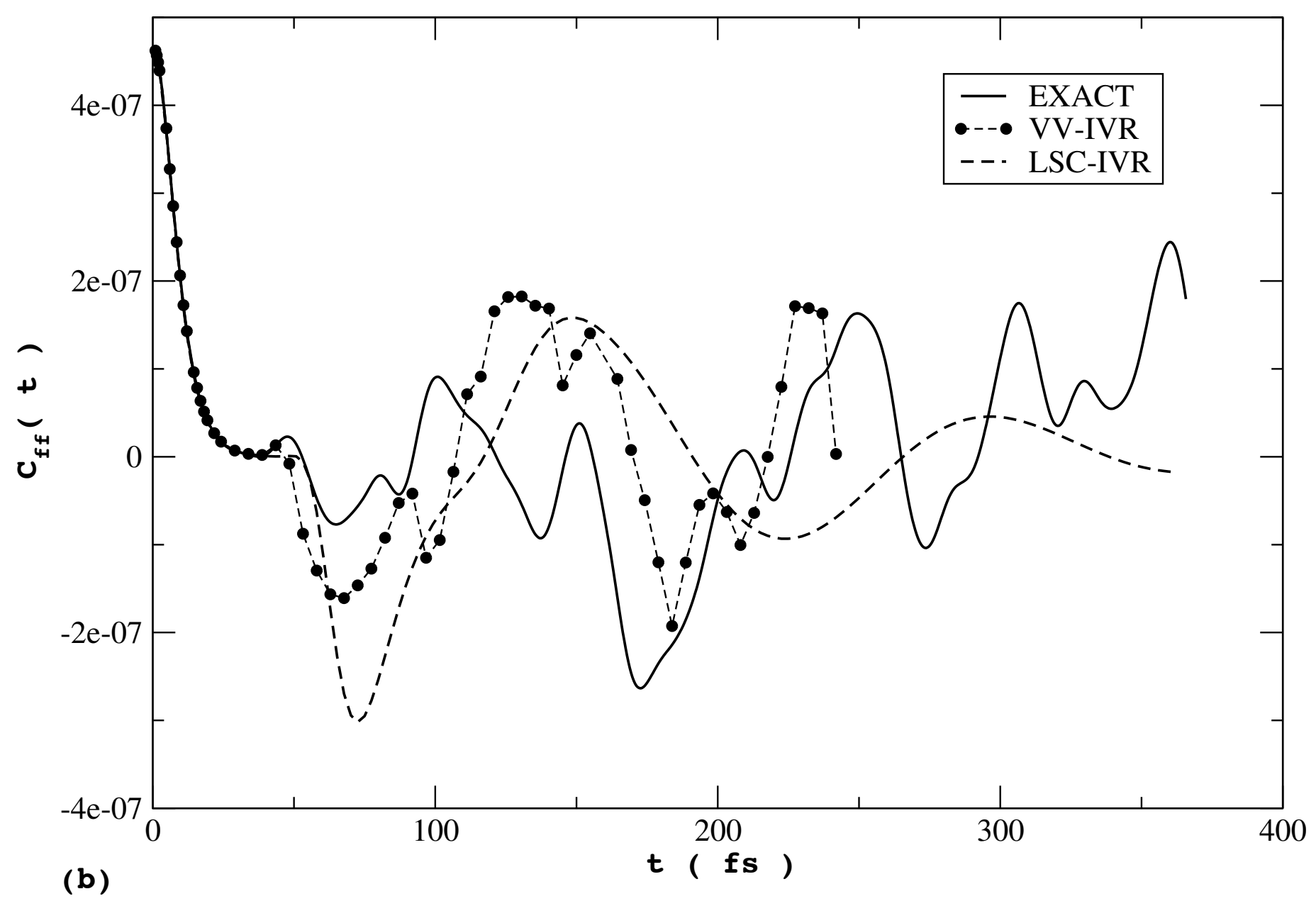




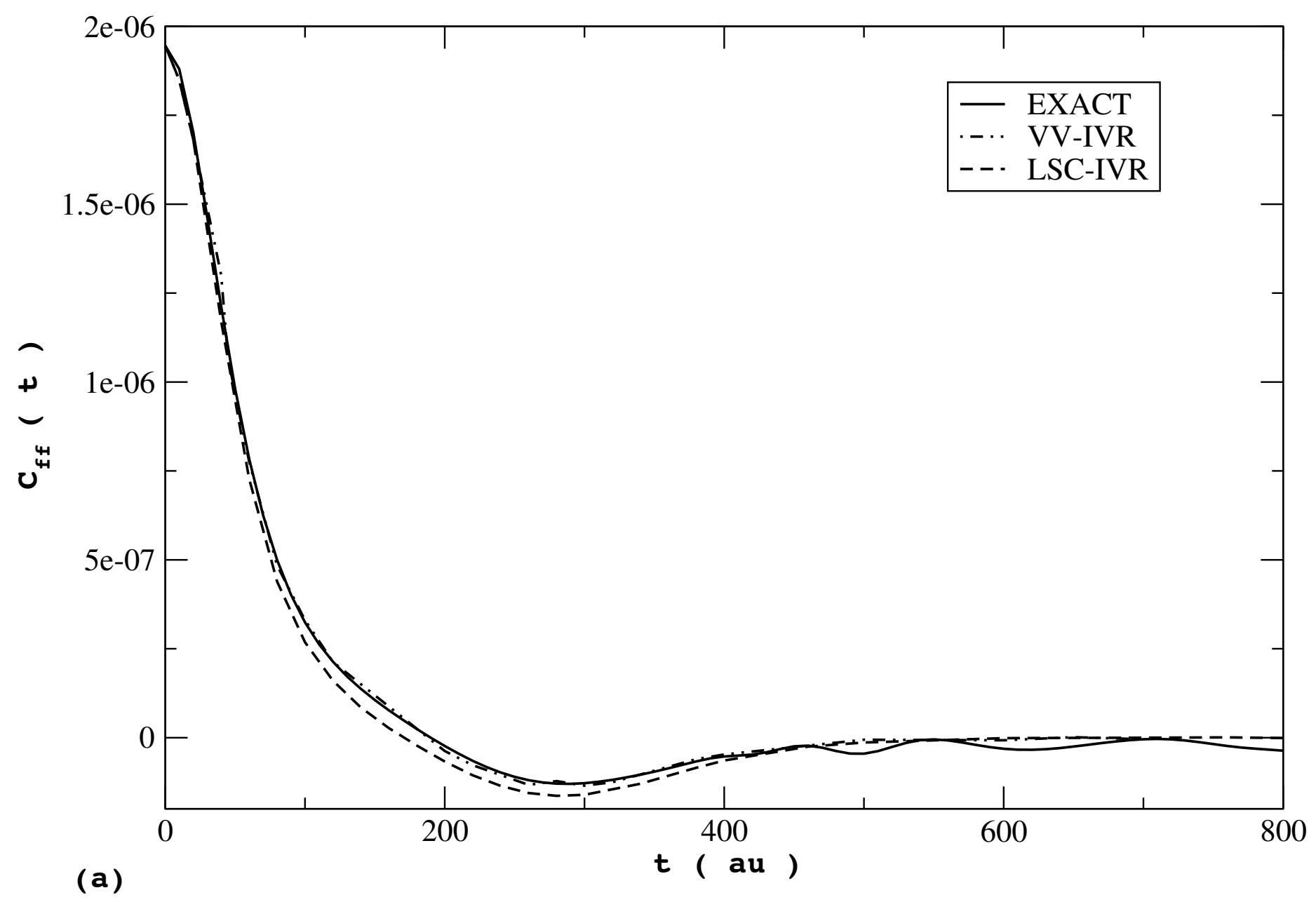




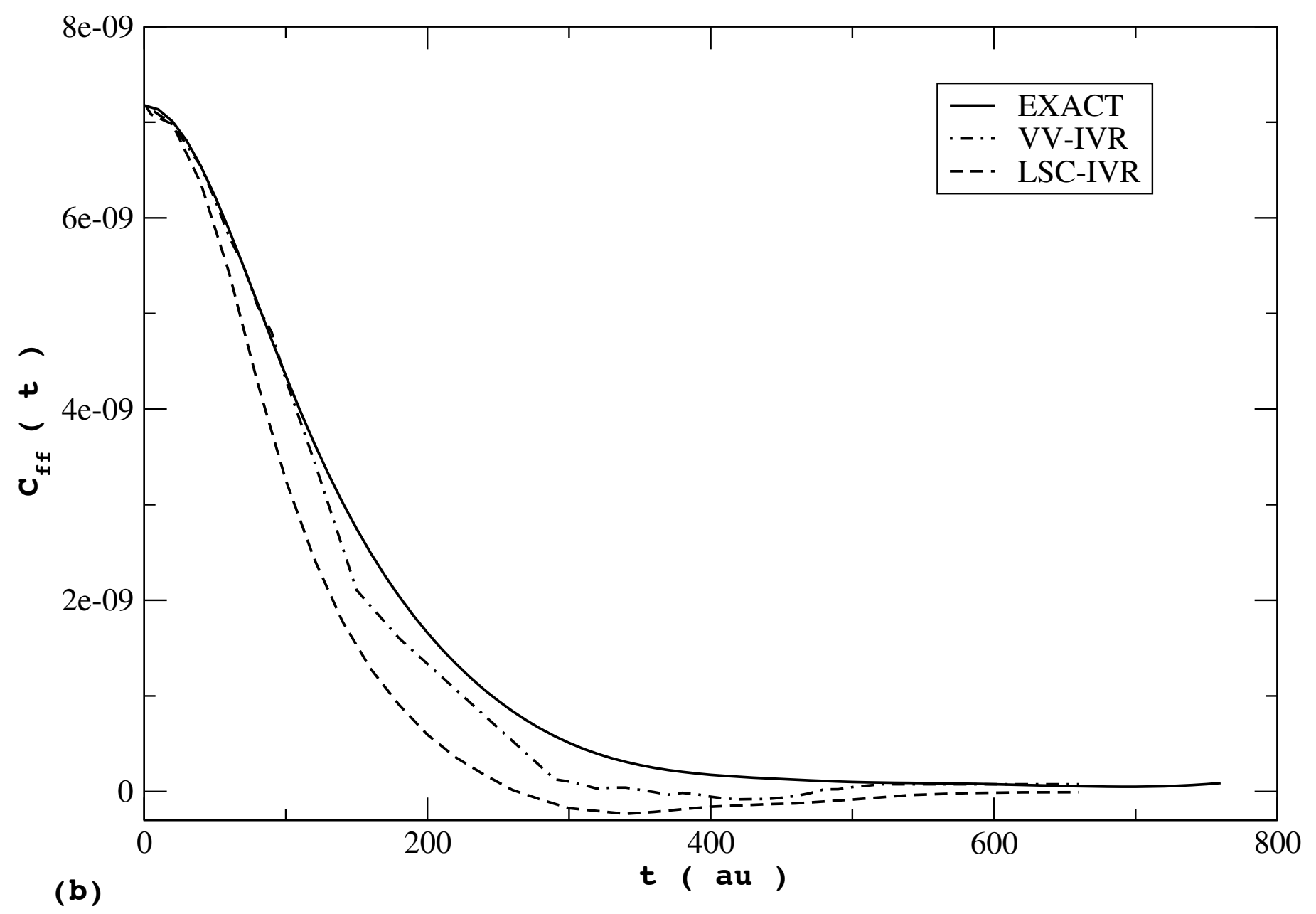




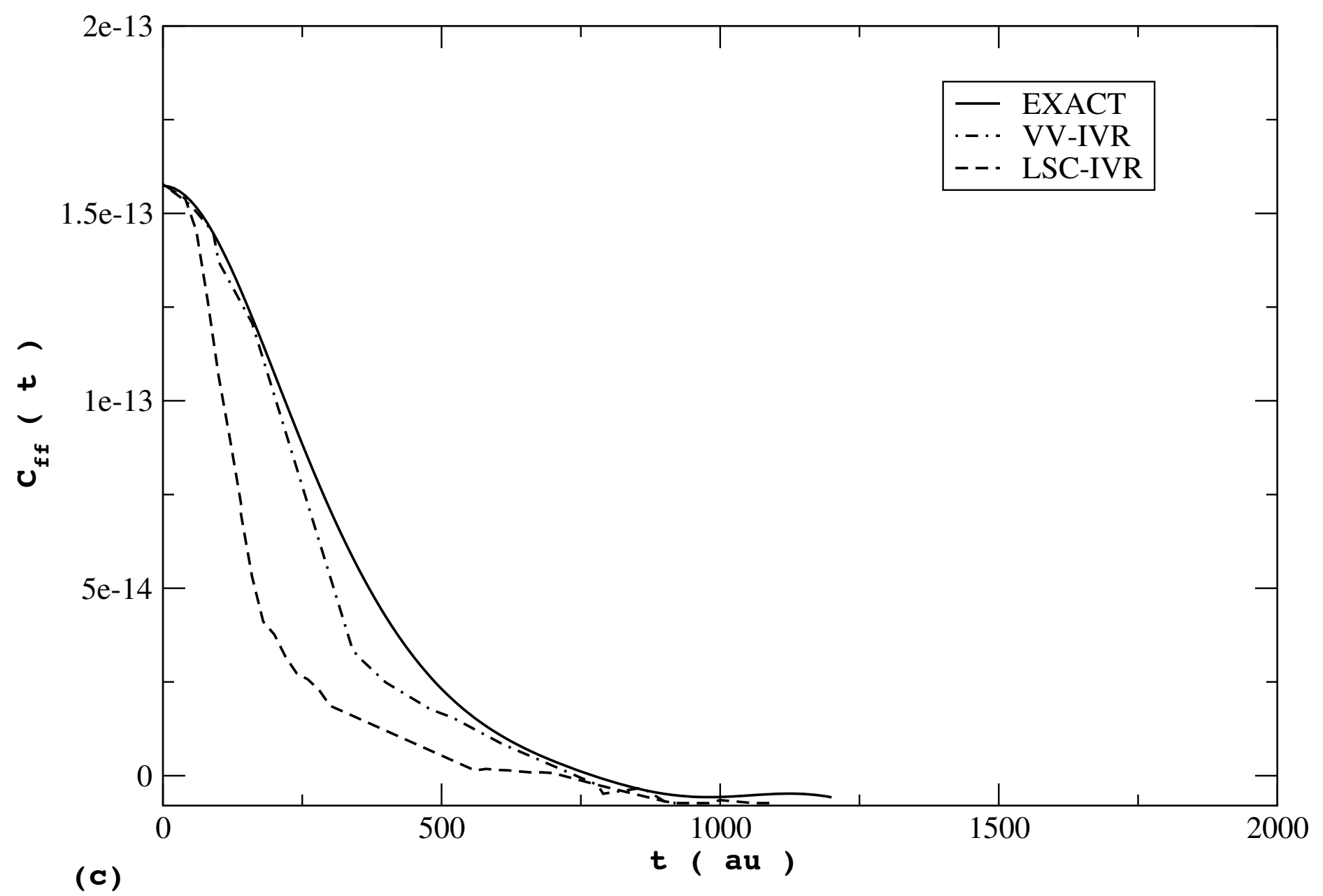




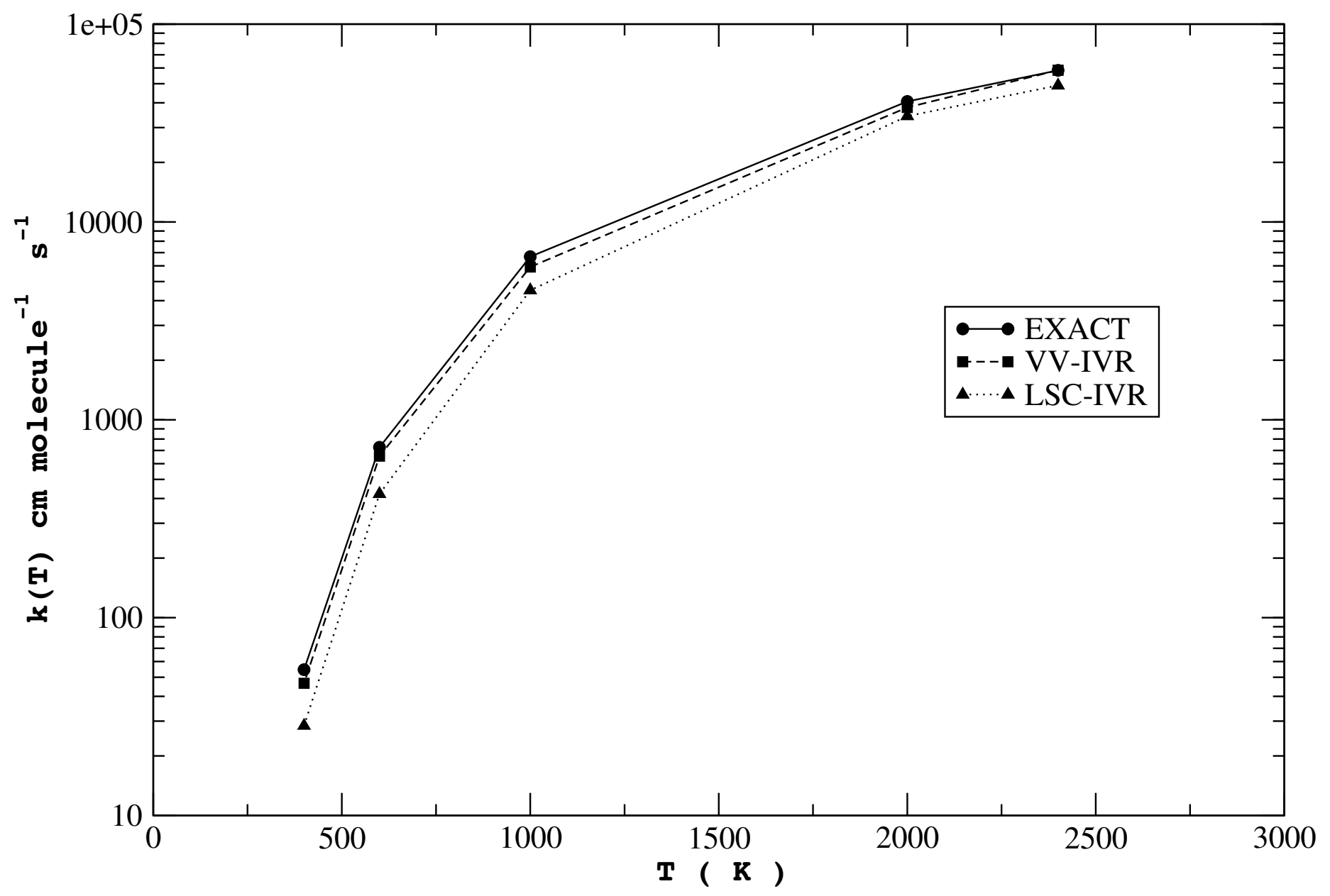

